\title{
Co-expression of herpes simplex virus thymidine kinase and Escherichia coli nitroreductase by an hTERT-driven adenovirus vector in breast cancer cells results in additive anti-tumor effects
}

\author{
BIN YU ${ }^{1,2^{*}}, Y_{U} Z_{H A N G}^{1,2^{*}}, Y_{A N G} Z H_{A N}^{1,2}$, XIAO ZHA ${ }^{3}$, YONEGE WU $^{1,2}$, \\ XIZHEN ZHANG ${ }^{1}$, QINGZHE DONG ${ }^{1}$, WEI KONG ${ }^{1,2}$ and XIANGHUI YU ${ }^{1,2}$ \\ ${ }^{1}$ National Engineering Laboratory for AIDS Vaccine, ${ }^{2}$ Key Laboratory for Molecular Enzymology and \\ Engineering, the Ministry of Education, College of Life Science, Jilin University, Changchun 130012; \\ ${ }^{3}$ Department of Molecular Pharmacology, Sichuan Tumor Hospital, Chengdu 610041, P.R. China
}

Received January 21, 2011; Accepted March 1, 2011

DOI: $10.3892 /$ or.2011.1285

\begin{abstract}
Breast cancers especially in the late and metastatic stages remain refractory to treatment despite advances in surgical techniques and chemotherapy. Tumor-specific promoter-directed suicide gene therapy and adenoviral technology can be promising strategies for such advanced disease. Previous studies suggested that combining herpes simplex virus thymidine kinase (HSV-TK) and ganciclovir (GCV) with Escherichia coli nitroreductase (Coli.NTR) and 5-(azaridin-1-yl)-2, 4-dinitrobenzamide (CB1954) by a recombinant retrovirus delivery system resulted in a co-operative killing effect in vitro. We constructed a bicistronic adenovirus type 5 (Ad5)-based vector which co-expresses herpes HSV-TK and Coli.NTR under the control of the human telomerase reverse transcriptase (hTERT) promoter and SV40 enhancer. NTR gene expression mediated by an internal ribosome entry site (IRES) was inserted after the hTERT and HSV-TK sequences. Anti-tumor activities of the novel vector, Ad-hT-TK/NTR-enh, combined with prodrugs were evaluated in human breast cancer cells (ZR-75-30, MCF-7) in vitro and in vivo. We showed that expression of HSV-TK and NTR genes by Ad-hT-TK/NTR-enh in combination with GCV and
\end{abstract}

Correspondence to: Dr Wei Kong or Dr Xianghui Yu, College of Life Science, Jilin University, No. 2699 Qianjin Street, Changchun 130012, P.R. China

E-mail: weikong@mail.jlu.edu.cn

E-mail: xianghui@mail.jlu.edu.cn

${ }^{*}$ Contributed equally

Abbreviations: HSV-TK, herpes simplex virus thymidine kinase; Coli.NTR, Escherichia coli nitroreductase; IRES, internal ribosome entry site; GCV, ganciclovir; CB1954, 5-(azaridin-1-yl)-2, 4-dinitrobenzamide; hTERT, human telomerase reverse transcriptase; MOI, multiplicity of infection; ANOVA, analysis of variance

Key words: HSV-TK, Escherichia coli nitroreductase, adenovirus, breast cancer, hTERT, internal ribosome entry site
CB1954 resulted in specific and significant cytotoxic effects in breast cancer cells in vitro. The anti-tumor activity of this system was more efficient than that from a single suicide gene, and only slightly lower than by HSV-TK and NTR driven from separate hTERT promoters in vitro and in vivo while the total amount of adenovirus of Ad-hT-TK/NTR-enh was half that of Ad-hT-TK-enh+Ad-hT-NTR-enh. These results suggest that suicide genes HSV-TK and NTR mediated by a single adenovirus vector under the control of an enhanced hTERT promoter results in additive anti-tumor effects and may provide a relatively safe strategy for the treatment of breast cancer by tumor-specific targeting.

\section{Introduction}

Breast cancer is the most common type of cancer and the second leading cause of cancer death in American women (1). It comprises $10.4 \%$ of all cancers among women, making it the second most common type of non-skin cancer (after lung cancer) and the fifth most common cause of cancer death worldwide (2). Since there is currently no effective treatment for late stage and metastatic breast cancers, and cytotoxic chemotherapy offers only palliation usually accompanied with systemic toxicities and poor quality of life, novel treatment modalities are needed for patients with advanced disease (3).

Suicide gene therapy, alternatively known as gene directed enzyme prodrug therapy (GDEPT), targeting the cytotoxic effect to the tumor site is a promising approach for treatment of such advanced cancers. Killing of a zone of cells around gene-modified cells due to transfer of toxic metabolites, known as the bystander effect, also contributes to tumor regression (4). A vast range of enzyme prodrug combinations have been designed for use in enzyme prodrug therapy, but few have made it through to human clinical trials. HSV-TK/ GCV is the only system to have reached phase III human trials $(5,6)$. NTR/CB1954 system also has reached clinical trials and has shown a strong bystander effect. However, previous studies suggested that expression of one suicide gene, even when the concentration of the prodrug was maximal, could 
not completely prevent tumor growth, while better anti-tumor effects could be observed in tumor cells transfected with two suicide genes, even when the concentration of each drug was very low (7).

The adenoviral vector has been used as a transfer vehicle to introduce genes into cancer cells as it is more efficient than non-viral gene transfer methods (e.g. cationic polymer-DNA complexes) $(8,9)$. More recently, another advantage to the use of adenoviral vectors has also become apparent, with the confirmation that integrating viral vectors (i.e., retrovirus, lentivirus, possibly adeno-associated viruses) can cause insertional mutagenesis and cancer in animal models and in human clinical trial subjects (10-14). Previous studies showed that the adenoviral vector mediated herpes simplex virus thymidine kinase (HSV-TK) suicide gene combined with ganciclovir (GCV) treatment of oral squamous carcinoma, malignant glioma and colorectal cancer had yielded some promising results (15-17). Nevertheless, there are still limitations to current suicide gene therapy strategies, including inefficient transfer and non-specific expression of therapeutic genes in normal cells, causing toxicity to non-cancerous tissues.

Recently, we and other groups have shown that adenoviruses carrying the human telomerase reverse transcriptase (hTERT) promoter could target expression of therapeutic genes in human nasopharyngeal carcinoma cells and breast cancer cells $(18,19)$. Song et al reported that an adenovirus vector can maintain cancer cell specificity through the hTERT promoter and that the activated expression of HSV-TK can be increased by an SV40 enhancer (20). In this study, we developed a therapeutic strategy by combining HSV-TK/GCV and NTR/ CB1954 treatment strategies by expressing the suicide genes under the control of the hTERT promoter and the SV40 enhancer within a single replication-defective adenovirus 5 (rAd5) vector to specifically enhance the cytotoxic effects of the suicide genes. We showed that such a strategy improved anti-tumor effects against breast cancer and may potentially be developed as a safe and effective treatment for breast cancer.

\section{Materials and methods}

Cell lines and cell culture. The CCD-11lu (human normal lung fibroblast) and HEK293 (human kidney cells transformed with Ad5 DNA) were purchased from the American Type Culture Collection (Manassas, VA). ZR-75-30 (human breast carcinoma), MCF-7 (human breast carcinoma), Fadu (human nasopharyngeal carcinoma), KB (human epidermal carcinoma of the mouth), and Hela (human uterine cervical carcinoma) cells were obtained from the Cell bank of Chinese Academy of Science (Shanghai, China). All cell lines were cultured in the media specified below along with $10 \%$ fetal bovine serum (FBS) (Invitrogen, Carlsbad, CA), penicillin $(50 \mathrm{U} / \mathrm{ml})$, and streptomycin $(50 \mu \mathrm{g} / \mathrm{ml})$ in the presence of $5 \%$ $\mathrm{CO}_{2}$. The CCD-11lu line was cultured in minimum essential media (MEM). The ZR-75-30, MCF-7, Hela and KB cells were cultured in RPMI-1640 medium. The Fadu line was cultured in Dulbecco's modified Eagle's medium (DMEM) supplemented with 1X MEM with non-essential amino acids and sodium pyruvate. The HEK293 cells were cultured in DMEM.
Telomeric repeat amplification protocol (TRAP) assay. Telomerase was extracted from cells by TRAP-silver staining telomerase detection kit (KeyGEN, Nanjng, China) according to the manufacturer's instructions. In brief, $1 \times 10^{6}$ cells were resuspended in $200 \mu \mathrm{l}$ of ice-cold lysis buffer and incubated for $30 \mathrm{~min}$ on ice. After centrifugation of lysate at $12,000 \mathrm{x} \mathrm{g}$ for $30 \mathrm{~min}$ at $4^{\circ} \mathrm{C}$, the resulting supernatant was stored at $-80^{\circ} \mathrm{C}$. The protein concentration of each extract was determined by BCA protein assay kit (Thermo Scientific Pierce, IL). Two microliters of diluted extract (200 ng/ $\mu \mathrm{l})$ was added to $48 \mu \mathrm{l}$ of reaction mixture containing 10X TRAP buffer $(5 \mu \mathrm{l}), 50 \mathrm{X}$ deoxynucleotide triphosphates (dNTPs) mix $(1 \mu 1)$, TS primer $(1 \mu \mathrm{l}), \mathrm{CX}$ primer $(1 \mu \mathrm{l}), 5 \mathrm{U} / \mu \mathrm{l} \mathrm{Taq}$ polymerase $(0.4 \mu \mathrm{l})$, and diethylpyrocarbonate (DEPC) $\mathrm{H}_{2} \mathrm{O}$ $(39.6 \mu \mathrm{l})$. The tubes were incubated in thermocycler at $30^{\circ} \mathrm{C}$ for $30 \mathrm{~min}$ for the elongation of TS primer by telomerase and followed by 33 cycles of PCR amplification $\left(94^{\circ} \mathrm{C}\right.$ for $30 \mathrm{sec}$, $50^{\circ} \mathrm{C}$ for $30 \mathrm{sec}$ and $72^{\circ} \mathrm{C}$ for $60 \mathrm{sec}$ ). The PCR products were examined on non-denaturing polyacrylamide gel $(8 \% \mathrm{w} / \mathrm{v})$ in Tris-borate-EDTA (TBE) buffer and stained with the silver staining method.

Reverse transcription (RT)-PCR. Total RNA was isolated using the RNeasy mini kit (Qiagen, Hilden, Germany) and treated with DNaseI to avoid genomic DNA contamination. cDNA was synthesized from $2 \mu \mathrm{g}$ of RNA using the SuperScriptII reverse-transcriptase (Invitrogen) with Oligo(dT)15 primers. The hTR was amplified with hTR specific primers (5'-TTTGTCTAACCCTAACTGAGAAGG-3' as forward and 5'-TGTGAGCCGAGTCCTGGGTGCACG-3' as reverse primer). hTERT mRNA was amplified with hTERT specific primers (5'-CGGAAGAGTGTCTGGAGCAA-3' as forward and 5'-GGATGAAGCGGAGTCTGGA-3' as reverse primer). GAPDH was amplified as a positive control (5'-GCTGCTTT TAACTCTGGTAA-3' and 5'-AGACCACCTGGTGCTCAG-3' as reverse primer).

Flow cytometry (FCM). For detection of hTERT expression, cells were harvested by two centrifugations at $3,000 \mathrm{x} \mathrm{g}$ for $10 \mathrm{~min}$ at $4^{\circ} \mathrm{C}$. The cell pellets were resuspended in appropriate amount of pre-cooled PBS to make a suspension with cell density of $1 \times 10^{6}$ cells $/ \mathrm{ml}$. The cells were lysed with $1 \%$ Triton X-100 and incubated with primary antibody, antihTERT polyclonal antibody (Santa Cruz Biotechnology, Santa Cruz, CA) for $30 \mathrm{~min}$ at room temperature. The reaction mixture was then washed with PBS buffer three times for $15 \mathrm{~min}$ each and incubated at room temperature for $30 \mathrm{~min}$ with FITC-labeled anti-rabbit antibody (Dingguo, Beijing, China) in a darkroom. The cells were washed 3 times with PBS for $15 \mathrm{~min}$ each. FCM was performed to detect the expression of hTERT.

Construction of recombinant adenoviral vectors. The recombinant adenovirus plasmids Ad-CMV-TK-enh and Ad-hT-TK-enh were constructed as previously described $(18,20)$. The Escherichia coli nitroreductase (Coli.NTR; GenBank no. U07860; from 176 bp to 829 bp) gene was amplified by PCR using LA Taq DNA polymerase (Takara, Aichi, Japan) from the Top10 genomic DNA. The PCR product was cloned into the pGl3-SV40 enhancer vector at 
HindIII and $X b a \mathrm{I}$ sites to obtain pGl3-hT-NTR-enh. The human IRES sequence (GenBank no. NW001842395.2; from 450232 bp to 450884 bp) was amplified from the pIRES2EGFP (BD Biosciences, Clontech, Palo Alto, CA). The PCR products of the IRES and NTR gene were inserted by overlapping PCR into pGl3-hT-TK/NTR-enh. The expression cassettes of hT-NTR-enh and hT-TK/NTR-enh were amplified by PCR to introduce I-CeuI and PI-SceI sites and cloned into the replication-incompetent Ad5 DNA (Adeno-X Viral DNA, Clontech) to obtain Ad-hT-NTR-enh and Ad-hT-TK/ NTR-enh. The constructed adenoviral vectors were digested with $\mathrm{PacI}$ and transfected into HEK293 cells. The rAd5 were propagated in HEK293 cells and purified by $\mathrm{CsCl}$ gradient centrifugation (21). The PFU titers were determined by the TCID $_{50}$ method. The negative control vector, Ad-Blank, is the adenovirus type 5 genome vector lacking the E1 and E3 regions (Newgene, Inc., Shanghai, China).

Luciferase activity assay. HEK 293 were plated at $5 \times 10^{5}$ cells per well in six-well plates and cultured overnight. Cells were transfected the following day with FuGENE ${ }^{\mathrm{TM}} 6$ transfection reagent (Roche, Penzberg, Germany) following the manufacturer's instructions. The pGl3-CMV-luc-enh $(0.8 \mu \mathrm{g})$ and pGl3-hTERT-luc-enh $(0.8 \mu \mathrm{g})$ were transfected into HEK293 cells. After $48 \mathrm{~h}$, luciferase activity was measured with a luciferase assay kit (Promega, Madison, WI). To determine the transfection efficiencies, the pDC316-EGFP vector was transfected into HEK293 cells under the same conditions, and the expression of green fluorescent protein (GFP) was observed using an inverted fluorescence microscope (data not shown).

Western blot analysis of protein expression. Cells were seeded at $5 \times 10^{5}$ cells per well in six-well plates. Twenty-four hours later, the cells were infected with recombinant adenoviruses at MOI of 50 and then harvested at $48 \mathrm{~h}$ post-infection. The cells lysates were suspended in a low salt extraction buffer, centrifuged $(3,000 \mathrm{rpm}$ for $5 \mathrm{~min})$, and the supernatants were collected as samples. Samples were mixed with an equal volume of $2 \mathrm{X}$ SDS buffer (125 mM Tris-HCl, $\mathrm{pH} 6.8$; 4\% SDS; $20 \%$ glycerol; $0.01 \%$ bromophenol blue; and $10 \%$ $\beta$-mercaptoethanol) and boiled for $30 \mathrm{~min}$. The samples were loaded onto a $12 \%$ polyacrylamide gel for SDS-polyacrylamide gel electrophoresis and then transferred to a nitrocellulose membrane. The primary antibodies used were rabbit antisera produced by our laboratory raised against full-length HSV-TK/NTR protein or a fragment of Knob (387-582 aa). The secondary antibody was AP-labeled anti-rabbit antibody (Jackson ImmunoResearch Laboratories, Inc, West Grove, PA).

MTT assay analysis of cytotoxic effects in vitro. Individual cell lines were plated into 96 -well plates at $3 \times 10^{3}$ cells/well. Twenty-four hours later, recombinant adenoviruses were applied at MOI of 10 . The cells were incubated at $37^{\circ} \mathrm{C}$ for $3 \mathrm{~h}$ followed by a change of media containing various concentrations of GCV. The cell cultures were replenished every day with fresh media containing GCV for the next two days. Various concentrations of CB1954 were added at the third media change containing GCV. After three changes of media with GCV, $20 \mu 1$ MTT $(5 \mathrm{mg} / \mathrm{ml})$ was added to each
A B
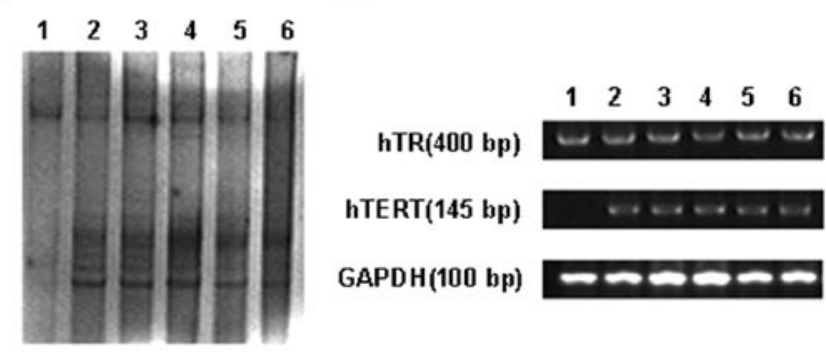

C
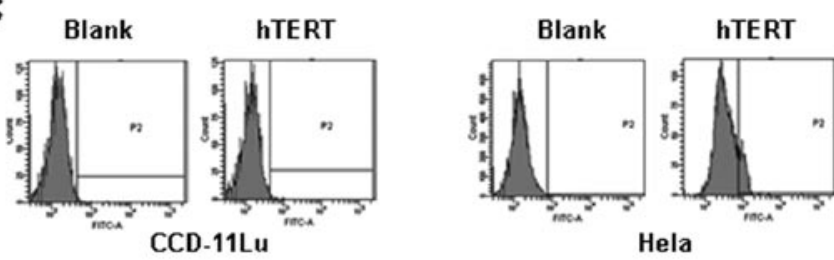

Hela
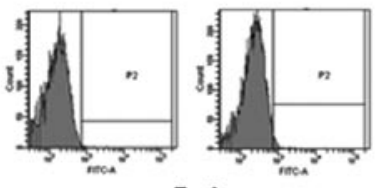

Fadu
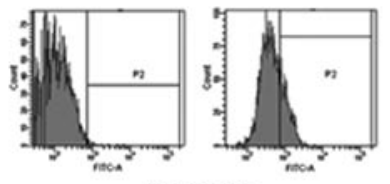

ZR-75-30

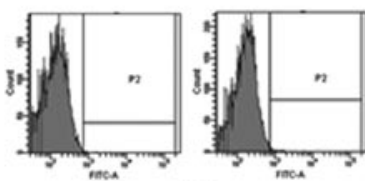

KB
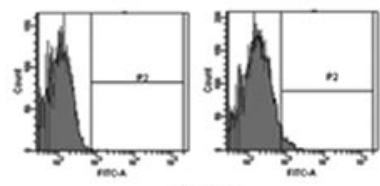

MCF7

Figure 1. Telomerase activity and hTERT expression in experimental cell lines. (A) Telomerase activity was assayed by TRAP assay using $2 \times 10^{6}$ cells. (B) The total RNA was isolated and used for RT-PCR in the presence of hTR, hTERT and GAPDH with specific primers. The PCR products were visualized by $1.0 \%$ agarose gel electrophoresis and ethidium bromide staining (1. CCD-11Lu; 2. Fadu; 3. KB; 4. ZR-75-30; 5. MCF-7; 6. Hela). (C) hTERT expression was assayed by flow cytometric analysis of $1 \times 10^{4}$ cells.

well, and the cells were incubated for another $4 \mathrm{~h}$. The MTT containing media was then discarded, and $150 \mu \mathrm{l}$ DMSO (control) was added to each well and vortexed for $10 \mathrm{~min}$ to dissolve the crystals. Absorbance (A) values were measured at $492 \mathrm{~nm}$ with a microplate reader. Cell proliferation rates were calculated according to the following formula: proliferation = [(average A value of experimental group - average A value of control group) / (average A value of positive group - average A value of control group)] x $100 \%$.

Evaluating anti-tumor effects in vivo by tumor growth assay. Six-week-old female athymic mice (BALB/c nu) were subcutaneously injected with $5 \times 10^{5}$ ZR-75-30 cells on day 1 . Adenovirus vectors $\left(1 \times 10^{8} \mathrm{PFU}\right.$, Ad-hT-TK-enh+AdhT-NTR-enh each at $\left.1 \times 10^{8} \mathrm{PFU}\right)$ in $50 \mu \mathrm{l}$ PBS was injected into the tumor foci on day $2, \mathrm{GCV}(3.75 \mathrm{mg} / \mathrm{kg})$ was injected intraperitoneally from days 4 to 8 , and CB1954 $(80 \mathrm{mg} /$ $\mathrm{kg}$ ) or sodium chloride was injected intraperitoneally on days 4 and 11. All mouse experiments were approved by the University Committee on the Use and Care of Animals of Jilin University. Tumor sizes were measured every 4 days from days 14 to 30 , and the volumes were calculated using the following equation: [(the longest diameter) $\mathrm{x}$ (the shortest

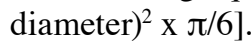


A

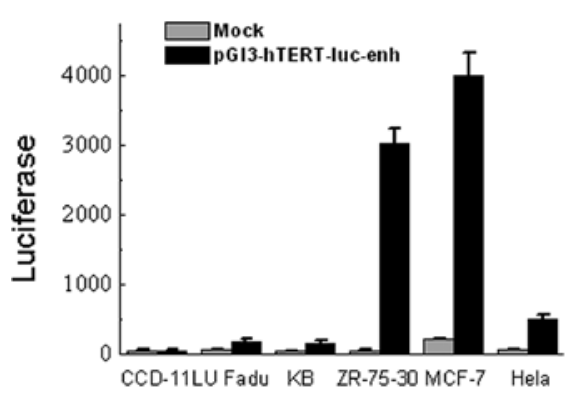

C

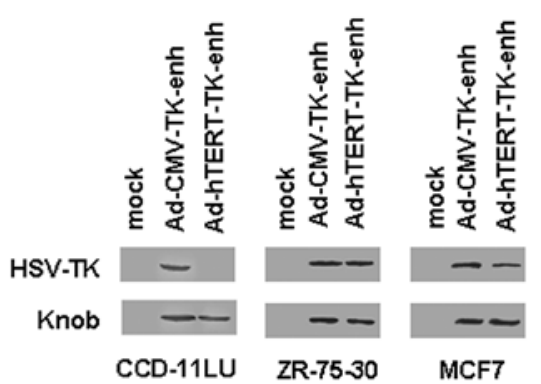

B

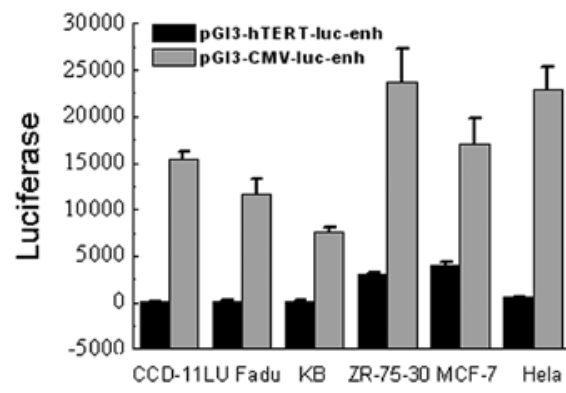

D

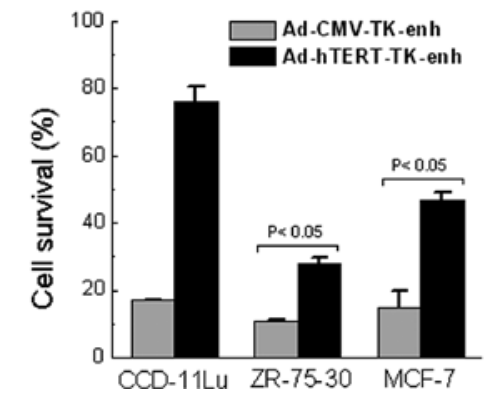

Figure 2. Comparisons of hTERT and CMV promoter activities, their protein expression and cytotoxic effects in tumor and normal cells. Luciferase activities were analyzed using the Promega luciferase assay system on experimental cell lines harvested $48 \mathrm{~h}$ after transient transfection with (A) pGl3-hTERT-luc-enh compared with Mock or (B) pGl3-hTERT-luc-enh compared with pGl3-CMV-luc-enh. Results from a representative experiment are shown as the means \pm SE from quadruplicate wells. (C and D) Protein expression and cytotoxic effects of proteins driven by hTERT were compared with that of a CMV promoter in the adenovirus vector in breast cancer and normal cells. (C) Western blot showing expression of HSV-TK proteins in Ad-CMV-TK-enh or Ad-hTERTTK-enh infected at MOI 50 in normal and breast cancer cells. The polyclonal primary antibody was raised against full-length HSV-TK, and a Knob fragment was detected as the loading control. (D) Cell survival of Ad-CMV-TK-enh and Ad-hTERT-TK-enh infected experimental cell lines with GCV treatment. Experimental cells $\left(3 \times 10^{3}\right)$ were seeded in 96 -well plates $24 \mathrm{~h}$ prior to infection with rAds at MOI of 10 , and the culture medium containing $100 \mu \mathrm{M}$ of GCV was replaced after $3 \mathrm{~h}$ and after 2 days with the same concentrations of GCV. The living cells were measured using an MTT assay 2 days after the last GCV treatment. Data were derived from averages of three independent experiments.

Evaluating specific activity of hTERT in vivo by RT-PCR. $\mathrm{BALB} / \mathrm{c}$ Nu mice were subcutaneously injected with $5 \times 10^{5}$ ZR-75-30 cells. rAds were injected into the tumor foci when the volume of the tumor was up to $30 \mathrm{~mm}^{3}$. The mice were sacrificed on day 3 after the injection. Tumor and adjacent normal tissues were polished, and the total RNA was isolated using the RNeasy mini kit (Qiagen) and treated with DNaseI to avoid genomic DNA contamination. The total RNA was then used for RT-PCR analysis of the presence of HSV-TK, NTR and E2b with specific primers. The PCR products were visualized after $1.0 \%$ agarose gel electrophoresis and ethidium bromide staining.

Statistical analysis. ANOVA (i.e. one-way ANOVA followed by Fisher's LSD) was employed using SPSS 13.0 software. $\mathrm{P}<0.05$ was considered to represent a significant difference. All data points are presented as means \pm SE (standard error).

\section{Results}

Expression and activity of telomerase in cancer cell lines. To determine the levels of telomerase in experimental cells, telomerase activity was assayed by the TRAP assay. As expected, breast carcinoma cell lines and other cancer cells showed strong telomerase activity, but normal cells did not (CCD-11Lu; Fig. 1A). To confirm the results of the TRAP assay, we analyzed hTERT expression by RT-PCR and flow cytometry. The results were consistent with the results of the TRAP assay: hTR expression was detected in all cell lines, while hTERT was detected only in cancer cell lines but not in normal fibroblast CCD-11Lu (Fig. 1B and C). These results revealed there was a close relation between the telomerase activity and hTERT expression levels in the experimental cell lines.

Activity of hTERT promoter in non-viral and adenoviral vectors in vitro. Before constructing the hTERT promoterdriven bicistronic adenovirus vector, we examined whether the hTERT promoter could enhance transcriptional activity in our breast carcinoma cell lines compared with normal cells. We first tested the specificity and activity of the hTERT promoter compared with the CMV promoter in a plasmid DNA vector (pGl3-SV40 enhancer). As shown in Fig. 1A, the hTERT promoter was 60 - and 80 -fold more active in breast tumor ZR-75-30 and MCF-7 cells, respectively, compared with normal human cells (CCD-11Lu). Moreover, the activity of the hTERT promoter in these breast cancer cell lines was even much more active than in three other cancer cell lines (Fadu, KB and Hela). Although the CMV promoter was 7.6- and 4.8-fold more active than the hTERT promoter in ZR-75-30 and MCF-7 cells, respectively, it was 300-fold more active than the hTERT promoter in CCD-11Lu normal cells (Fig. 2A and B) $(\mathrm{P}<0.01)$. 
A

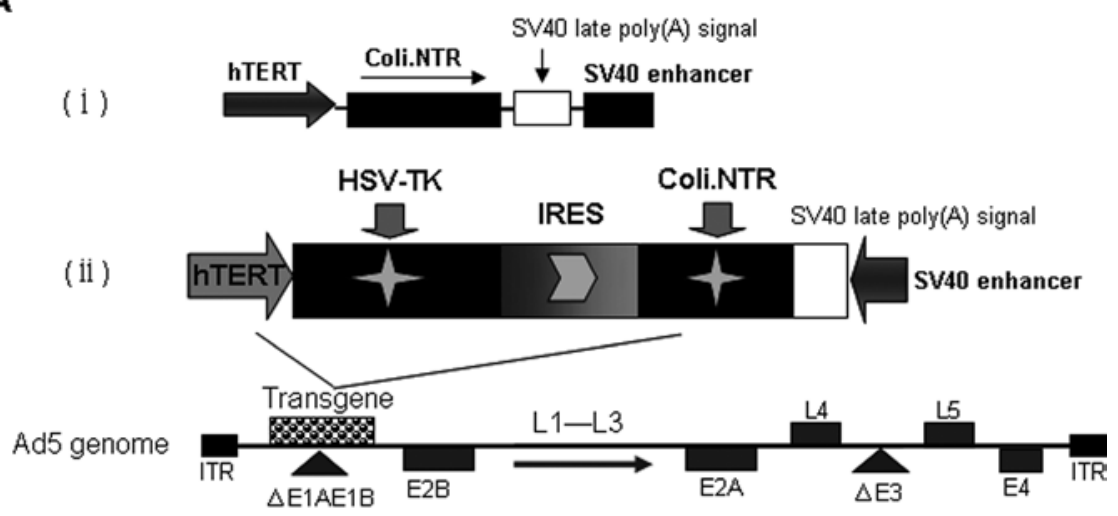

$\mathbf{B}$

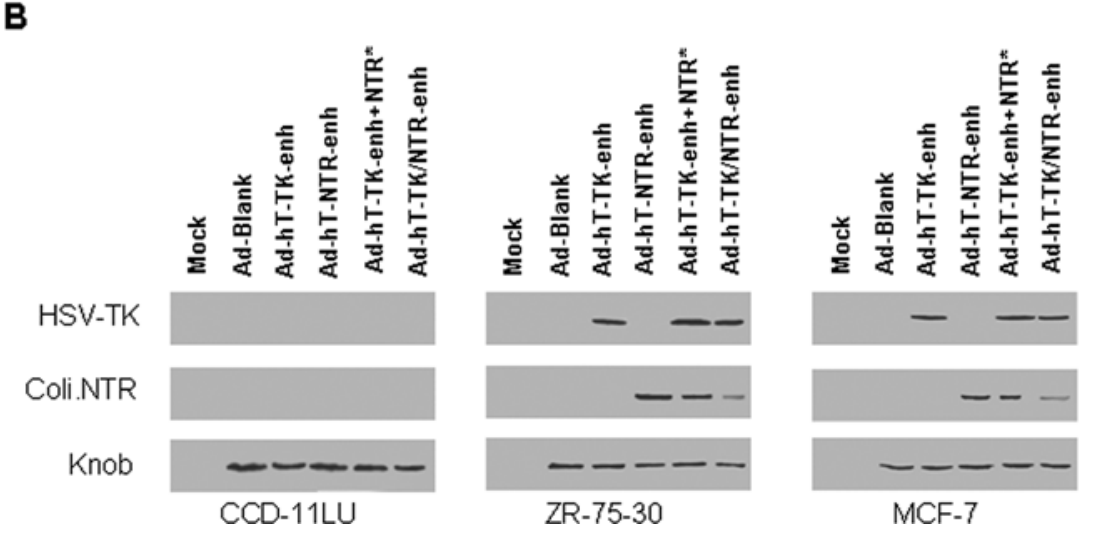

Figure 3. Schematic structure of recombinant Ad-hT-TK/NTR-enh and its expression of HSV-TK and NTR proteins in cancer and normal cells. (A) The (ii) elements are the bicistronic region which replaced the E1A region. The rest of the genome is similar to wild-type Ad5 except for the deletion of the E3 region. (B) Western blot showing expression of HSV-TK, NTR and Knob protein by rAd5 (Ad-hT-TK-enh, Ad-hT-NTR-enh, Ad-hT-TK-enh+Ad-hT-NTR-enh or Ad-hT-TK/NTR-enh) infected normal and cancer cell lines. Experimental cell lines were infected with rAds at an MOI of 50 (Ad-hT-TK-enh+Ad-hTNTR-enh each at MOI of 50), incubated for $48 \mathrm{~h}$, and then analyzed by Western blot to detect HSV-TK, Coli.NTR and Knob expression. Data from three independent experiments were analyzed. Ad-hT-TK-enh + NTR* = Ad-hT-TK-enh+Ad-hT-NTR-enh.

We then evaluated the protein expression and cytotoxic effect of the hTERT promoter in an adenovirus vector using two rAd5 based vectors (Ad-CMV-TK-enh and Ad-hTTK-enh) constructed previously in our laboratory. Western blotting showed that HSV-TK was expressed in the breast cancer cell lines tested in the study after infection with Ad-CMV-TK-enh and Ad-hT-TK-enh. By contrast, expression of HSV-TK was only detected in Ad-CMV-TK-enh in the normal fibroblast cell line CCD-11Lu (Fig. 2C). After adding GCV, cellular proliferation was evaluated by the MTT assay. As shown in Fig. 2D, after infection with Ad-hT-TK-enh, 78\% of normal cells (CCD-11Lu) were still alive even at $100 \mu \mathrm{M}$ GCV, while only 28\% of ZR-75-30 and 48\% of MCF-7 were alive. However, only 11-20\% of experimental cells were alive in all groups of Ad-CMV-TK-enh treated with GCV. These results demonstrate that adenovirus vectors with the hTERT promoter could specifically express therapeutic genes in human breast cancer cells and significantly decrease the viability of ZR-75-30 and MCF-7 cells in vitro. We also showed that the Ad-hT-TK-enh/GCV system was significantly less effective against tumor cells compared with the Ad-CMV-TK-enh/GCV system in vitro $(\mathrm{P}<0.05)$.

Construction of hTERT-driven bicistronic adenovirus and tumor-specific expression of HSV-TK and NTR proteins. To improve upon a gene therapy vector for treatment of breast cancer, we constructed a novel recombinant adenovirus, Ad-hT-TK/NTR-enh. We also constructed Ad-hT-NTR-enh as one of the control vectors (Fig. 3A). To assess the expression of HSV-TK and NTR under the control of the hTERT promoter and internal ribosomal entry site (IRES), cells were infected with Ad-hT-TK-enh, Ad-hT-NTR-enh, Ad-hT-TK-enh+AdhT-NTR-enh or Ad-hT-TK/NTR-enh. Western blot analysis showed that the level of HSV-TK protein expression in each cancer cell line was similar. Expression of NTR in each cancer cell line infected by Ad-hT-NTR-enh was considered as $100 \%$. Compared with Ad-hT-NTR-enh, expression of NTR in cancer cells infected with Ad-hT-TK-enh+Ad-hT-NTR-enh was almost equal, but only 10-20\% NTR expression in each cancer cell line infected with Ad-hT-TK/NTR-enh was observed (Fig. 3B). These results demonstrated that while the IRES primed translation of the NTR transcript in the cancer cells, the resulting NTR protein levels were lower than that from the hTERT promoter. By contrast, there were no significant levels of HSV-TK or NTR expressed in the hTERT negative normal lung fibroblast cell line (CCD-11Lu).

Analysis of cytotoxic effects in vitro by Ad-hT-TK/NTR-enh in combination with $G C V$ and CB1954. To assess the in vitro cytotoxic effect of our vectors, cells plated in 96-well plates were infected at MOI of 10 as described in Materials and methods. Culture media containing $\operatorname{GCV}(0.1,1,10$, or 
CCD-11Lu

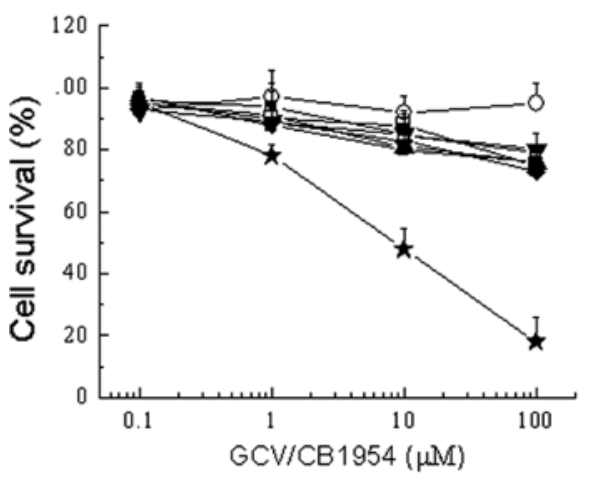

MCF-7

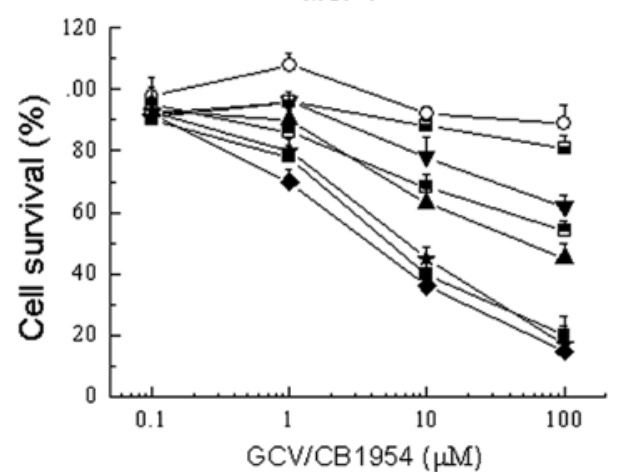

ZR-75-30

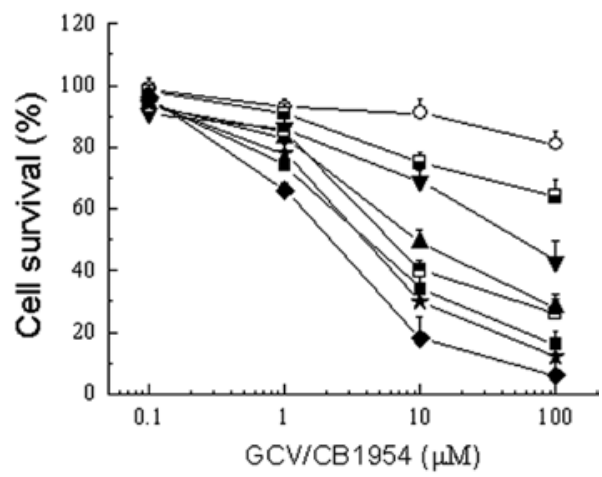

Figure 4. Cytotoxic effect in vitro by Ad-hT-TK/NTR-enh in combination with GCV and CB1954. Experimental cells (3x10 ${ }^{3}$ ) were seeded in 96-well plates $24 \mathrm{~h}$ prior to infection with rAd5 (Ad-hT-TK-enh, Ad-hT-NTR-enh, Ad-hT-TK-enh+Ad-hT-NTR-enh or Ad-hT-TK\NTR-enh) at MOI of 10 (Ad-hT-TK-enh +Ad-hT-NTR-enh each at MOI of 10). Concentrations of GCV and CB1954 were from 0.1 to $100 \mu \mathrm{M}$. The living cells were measured 2 days after the last prodrug change using the MTT assay. Each experiment was performed in triplicate.

$100 \mu \mathrm{M})$ were replaced after $3 \mathrm{~h}$. Two days later, the cultures were replenished again with media containing GCV at the same concentrations. CB1954 at various concentrations $(0.1,1$, 10 , or $100 \mu \mathrm{M})$ was added in culture media at the last media change. The living cells were measured 2 days after the last media change using the MTT assay.

As shown in Fig. 4, the two types of double suicide gene systems (Ad-hT-TK/NTR-enh/GCV+CB1954 and Ad-hT-TKenh+Ad-hT-NTR-enh/GCV+CB1954) and Ad-CMV-TK-enh/ GCV groups resulted in greater cytotoxic effects and significantly better inhibition of tumor growth when compared to Ad-blank/GCV+CB1954 at 10 and $100 \mu \mathrm{M}$ in breast cancer cells $(\mathrm{P}<0.01)$. When the concentration of prodrugs was at $100 \mu \mathrm{M}$, cell survival rates of Ad-hT-TK/NTR-enh/ $\mathrm{GCV}+\mathrm{CB} 1954$ group were 16\% in ZR-75-30 cells and 20\% in MCF-7 cells. Meanwhile in the Ad-hT-TK-enh/GCV group and Ad-hT-NTR-enh/CB1954 group the survival rates were significantly increased in ZR-75-30 cells (28 and 43\%) and MCF-7 cells (46 and 64\%) $(\mathrm{P}<0.05)$. When Ad-hT-TK/ NTR-enh combining separately with either GCV or CB1954, cell survival rates were $26 \%$ or $64 \%$ in ZR-75-30 cells and $54 \%$ or $82 \%$ in MCF-7 cells, respectively, with GCV or CB1954 at $100 \mu \mathrm{M}$. Furthermore, in CCD-11Lu normal cells only the Ad-CMV-TK-enh/GCV group showed a significant cytotoxic effect. These results indicated that Ad-hT-TK/NTR-enh could confer sensitivity to GCV and CB1954 in breast cancer cells but did not affect the normal lung fibroblast cell line. Also, the Ad-hT-TK/NTR-enh/GCV+CB1954 system resulted in additive anti-tumor effects in vitro.

Ad-hT-TK/NTR-enh combined with GCV+CB1954 treatment significantly inhibited the growth of breast tumor cells in vivo. To confirm the results of the MTT assay from the in vitro studies and further establish the Ad-hT-TK/NTR-enh/ $\mathrm{GCV}+\mathrm{CB} 1954$ treatment as an effective adenoviral therapy against breast cancer, we implanted $5 \times 10^{5}$ ZR-75-30 cells in the right hind limbs of $\mathrm{BalB} / \mathrm{C} \mathrm{Nu}$ mice. Vectors and prodrugs were injected as described in Materials and methods and as shown in Fig. 5C. The groups of vectors in combination with prodrugs are shown in Table I. The sizes of the tumors were measured every 4 days. The results showed that from day 22 to 30, the Ad-hT-TK/NTR-enh/GCV+CB1954 group had significantly inhibited growth of tumor cells compared with the PBS group and Ad-blank group $(\mathrm{P}<0.01)$, the results of which were superior to the Ad-hT-TK-enh/GCV and Ad-hT-NTR-enh/CB1954 treatments $(\mathrm{P}<0.05)$ (Fig. 5A). In Fig. 5B, the results indicated that the anti-tumor activity of the Ad-hT-TK/NTR-enh/GCV+CB1954 group was close to that of the Ad-CMV-TK-enh/GCV group and slightly lower although not statistically significant different $(\mathrm{P}>0.05)$ than the Ad-hT-TK-enh+Ad-hT-NTR-enh-/GCV+CB1954 group. To further study the effect of co-expressing double suicide genes with each prodrug in vivo, we combined Ad-hT-TK/NTR-enh with either GCV or CB1954 and found that compared with 
A

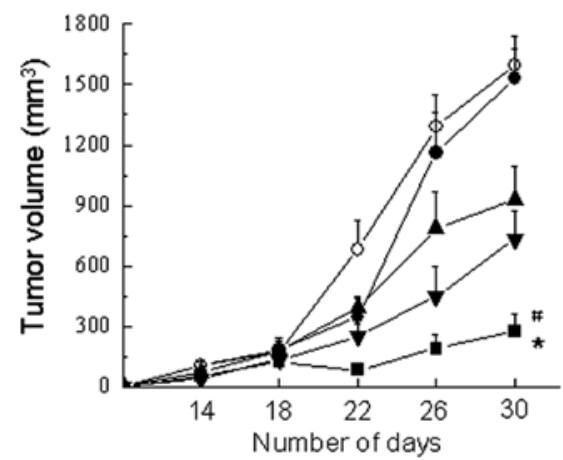

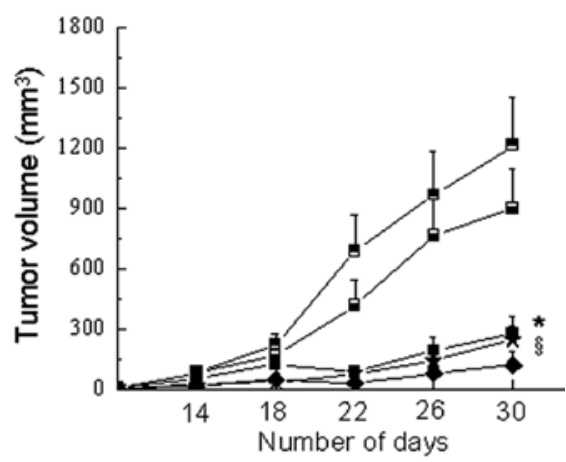

C

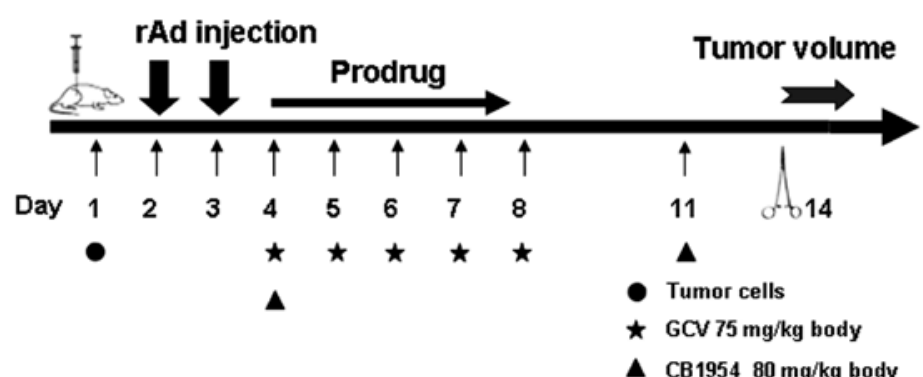

Figure 5. Growth curves of subcutaneous ZR-75-30 tumors in nude mice. The tumor growths of various groups were compared with that of the Ad-hT-TK/ NTR-enh/GCV+CB1954 group using ANOVA from days 22 to $30(n=5)$. (A) Compared with the PBS/sodium chloride group or Ad-blank/GCV+CB1954 groups, (\#) P<0.01; compared with the Ad-hT-TK-enh/GCV or Ad-hT-NTR-enh/CB1954 groups, ( $\star$ ) $\mathrm{P}<0.05$. (B) Compared with Ad-hT-TK/NTR-enh separately with GCV or CB1954, ( $\star$ P P $<0.05$; compared with Ad-CMV-TK-enh/GCV group or Ad-hT-TK-enh+Ad-hT-NTR-enh/GCV+CB1954, (§) P $>0.05$. (C) Design of the in vivo assay as described in Materials and methods.

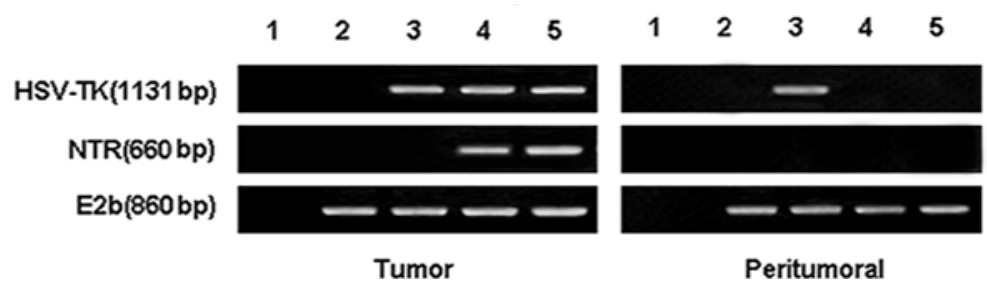

Figure 6. Specific expression of HSV-TK and NTR in vivo. E2b gene is shown as the loading control (1. mock, 2. Ad-blank, 3. Ad-CMV-TK-enh, 4. Ad-hT-TK/ NTR-enh, 5. Ad-hT-TK-enh+Ad-hT-NTR-enh) $(\mathrm{n}=3)$.

the Ad-hT-TK/NTR-enh/GCV+CB1954 group, the anti-tumor effects of the two treatments were significantly reduced $(\mathrm{P}<0.05)$. These results were consistent with the results of the MTT assay.

Tumor-specific gene expression of the hTERT promoterdriven Ad5 based vector. To examine whether the enhanced hTERT promoter-driven rAd5 based vector could specifically express genes in tumor tissues in vivo at the dose chosen for the study $\left(2 \times 10^{8} \mathrm{PFU}\right)$, we evaluated the expression of the HSV-TK and NTR genes in tumor and adjacent normal tissues by reverse transcription polymerase chain reaction (RT-PCR). As shown in Fig. 6, HSV-TK and NTR mRNA were detected in tumor cells after injection with the CMV promoter or hTERT promoter-driven adenovirus vectors. However, in the tumor adjacent normal tissues, only HSV-TK mRNA was detected in the Ad-CMV-TK-enh injected group. These results demonstrated that our bicistronic adenovirus vector could express suicide genes in the tumor but not in adjacent normal tissues, and there was no promoter interference within this single-vector platform.

\section{Discussion}

In an attempt to address the limitations of conventional chemotherapy, current research is focused on novel approaches such as suicide gene therapy for common and advanced cancers. Patients with glioblastoma multiforme have been treated with an HSV-TK-expressing replication defective retrovirus followed by GCV and radiotherapy (6), but this treatment failed to improve patient survival over standard chemotherapy (surgery and radiotherapy). However, a subsequent phase II trial that utilized a replication defective adenovirus for delivery of HSV-TK into patients with glioma produced a clinically and statistically significant increase in median survival from 38 to 62 weeks over standard therapy (5).

Nevertheless, clinically relevant tumor regressions continue to be only sporadic, and the limited biopsy data 
Table I. Vector and prodrug treatment combinations of mice groups.

\begin{tabular}{llll}
\hline Group & \multicolumn{1}{c}{ Map sign } & \multicolumn{1}{c}{ Intratumoral injection } & Intraperitoneal injection \\
\hline 0 & $\circ$ & PBS & Sodium chloride \\
1 & $\bullet$ & Ad-Blank & GCV+CB1954 \\
2 & $\star$ & Ad-CMV-TK-enh & GCV \\
3 & - & Ad-hT-TK-enh & GCV \\
4 & - & Ad-hT-NTR-enh & CB1954 \\
5 & Ad-hT-TK-enh+Ad-hT-NTR-enh & GCV+CB1954 \\
6 & Square with top half black & Ad-hT-TK/NTR-enh & GCV \\
7 & Square with bottom half black & Ad-hT-TK/NTR-enh & CB1954 \\
8 & - & Ad-hT-TK/NTR-enh & GCV+CB1954 \\
\hline
\end{tabular}

gathered during clinical trials suggest transgene expression is still a major limitation (22-24). Another clinically investigated enzyme prodrug therapy pair is Coli.NTR with CB1954. In vivo a stronger effect was reported enabling CB1954 to produce a significant growth delay in tumours containing only 5\% NTR-expressing cells (25). However, many studies noted that high doses of CB1954 administered to mice caused toxicity, mostly in the form of diarrhea due to the presence of NTR-expressing bacteria in the intestines. It has been reported that these two suicide genes, HSV-TK and NTR, may have addictive effects in anti-tumor responses. Bridgewater et al used a recombinant retrovirus to deliver the NTR gene or HSV-TK gene to murine and human cells in vitro, respectively, and demonstrated the addictive effects of CB1954 and GCV in NTR and TK-co-expressing cells (26).

A suicide gene strategy can be achieved by combining the targeted and efficient delivery of a gene vector with a tissue, tumor, or condition specific inducible promoter to restrict transgene expression to the tumor mass (27). Therefore, gene promoters, specifically those expressed in tumors may be utilized to effectively activate suicide gene vectors when the therapeutic gene expression is confined to the specific tumor tissue, minimizing secondary effects and maximizing therapeutic effects. Numerous studies have demonstrated that telomerase is activated in $>90 \%$ of malignant tumors, but it is stringently repressed in normal somatic cells. Telomerase activity is positively correlated with the tumor grade, and a strong correlation was found between hTERT expression and telomerase activity in a variety of tumors $(28,29)$. Therefore, the hTERT promoter has been used to induce tumor-specific trans-gene expression in cancer gene therapy using a single suicide gene to treat lung cancer, ovarian cancer, hepatocellular carcinoma, colon carcinoma, and nasopharyngeal carcinoma (30-33).

In the present study, we assessed the telomerase activity of two human breast cancer cell lines (ZR-75-30, MCF-7) by the TRAP assay and analyzed hTERT expression by RT-PCR and flow cytometry (FCM). The results demonstrated that breast cancer cell lines and other cancer cells showed strong telomerase activity and hTERT expression, but normal cells (CCD-11Lu) did not. hTERT expression was more apparent and resulted in more potent activity in ZR-75-30 cells than in other experimental cells, including two human head and neck cancer cells (Fig. 1). Compared with the CMV promoter, the hTERT promoter induced specific trans-gene expression and anti-tumor effects in breast cancer cells but not in normal cells in vitro and in vivo. However, an important point to be noted is that the hTERT promoter displayed specific activity in telomerase-positive cancer cells, but its promoter activity, being lower than that of the CMV promoter, induced insufficient anti-tumor activity (Fig. 2) although the SV40 enhancer was added to increase the activity of the hTERT promoter originally (20).

We also found that the SV40 enhancer could indeed increase the activity of the CMV promoter (data not shown). Thus, we used Ad-CMV-TK-enh with GCV as a reference treatment in anti-tumor assays in vitro and in vivo. In order to obtain a vector with tumor-specific targeting and relatively greater anti-tumor activity than Ad-CMV-TK-enh for treatment of breast cancer, we chose to express HSV-TK and NTR under the control of the hTERT promoter and SV40 enhancer. Furthermore, in order to reduce the vector dose and facilitate good manufacturing practice (GMP) grade production, we used the IRES to link the TK and NTR genes to achieve co-expression of exogenous genes. Although the NTR/CB1954 has a strong bystander effect, and high doses of CB1954 administered to mice can cause toxicity, the protein expression level depends on the type of cell that is transduced as well as the presence of other genetic elements within the vector (34) such as the IRES and NTR inserted after the HSV-TK gene in the hTERT promoter-driven bicistronic adenovirus vector, Ad-hT-TK/NTR-enh.

When we compared the expression of NTR in cancer cells with Ad-hT-NTR-enh and Ad-hT-TK-enh+Ad-hT-NTR-enh, we found that only $10-20 \%$ NTR was observed. Despite the low expression of NTR, however, our data suggest that the anti-tumor activity of Ad-hT-TK/NTR-enh combined with GCV+CB1954 was superior to Ad-hT-TK-enh with GCV or Ad-hT-TK-enh with CB1954, as shown by the MTT assays and tumor growth curves after we implanted nude mice with ZR-75-30 cells. These additive effects support the proposed difference in mode of action of the two activated prodrugs. The cells must be in S-phase for GCV-triphosphate to be incorporated into DNA to cause cytotoxicity, whereas activated CB1954, which acts as a crosslinking agent does not require cell division to affect viability. Co-operation between 
the actions of CB1954 and GCV could also be exploited to achieve an improved bystander killing effect. Although a strong bystander effect of NTR/CB1954 system was expected, we tested the Ad-hT-TK-enh+Ad-hT-NTR-enh experimental group in order to determine how the anti-tumor activity from the combination of TK/GCV and NTR/CB1954 would change given that the expression of TK or NTR was not affected by other factors. We found that when combining with GCV and CB1954 there was no obvious difference in anti-tumor activity between Ad-hT-TK-enh+Ad-hT-NTR-enh and Ad-hT-TK/ NTR-enh in vitro and in vivo, although the total amount of adenovirus of Ad-hT-TK/NTR-enh was half that of Ad-hTTK-enh+Ad-hT-NTR-enh. These results were likely caused by the strong bystander effect of NTR/CB1954.

Moreover, while Ad-CMV-TK-enh induced significantly higher expression levels of HSV-TK mRNA expression both in tumor and adjacent normal tissues, the HSV-TK and NTR genes under the transcriptional control of the hTERT promoter could only be detected in tumor tissues. Additionally, we examined whether our bicistronic adenovirus and prodrug treatments were harmful to the main organs of the experimental mice, and the results of histopathological analysis of kidneys and livers showed no obvious difference between our novel system and the mock treatments (data not shown).

In summary, we demonstrated that the suicide genes HSV-TK and NTR mediated by a single adenovirus vector platform under the control of the hTERT promoter resulted in additive anti-tumor activities in breast cancer cells in vitro and in vivo. The hTERT promoter combined with an SV40 enhancer can be used to induce tumor-specific trans-gene expression in cancer gene therapy to reduce toxicity while enhancing the anti-tumor activity. In further studies, we are attempting to graft this bicistronic expression cassette to a tissue-specific oncolytic adenovirus. Then we may be able to provide a more desirable vector to treat cancer, particularly metastatic breast cancer, although further in vivo investigations with these systems will still need to be performed in the future.

\section{Acknowledgements}

This study was supported by National Nature Science Foundation of China (no. 30872396) and the Key Projects in the National Science \& Technology Pillar Program in the Eleventh Five-year Plan Period (Grant no. 2008ZX10001-012).

\section{References}

1. Jemal A, Siegel R, Ward E, Hao Y, Xu J, Murray T and Thuns MJ: Cancer statistics, 2008. CA Cancer J Clin 58: 71-96, 2008.

2. Bernard WS and Paul K: World Cancer Report. International Agency for Research on Cancer. Lyon, 2003.

3. Altaner C: Prodrug cancer gene therapy. Cancer Lett 270: 191-201, 2008

4. Freeman SM, Abboud CN, Whartenby KA, Packman $\mathrm{CH}$, Koeplin DS, Moolten FL and Abraham GN: The 'bystander effect': Tumor regression when a fraction of the tumor mass is genetically modified. Cancer Res 53: 5274-5283, 1993.

5. Immonen A, Vapalahti M, Tyynelä K, Hurskainen H, Sandmair A, Vanninen R, Langford G, Murray N and Ylä-Herttuala S: AdvHSV-tk gene therapy with intravenous ganciclovir improves survival in human malignant glioma: A randomised, controlled study. Mol Ther 10: 967-972, 2004.
6. Rainov NG: A phase III clinical evaluation of herpes simplex virus type 1 thymidine kinase and ganciclovir gene therapy as an adjuvant to surgical resection and radiation in adults with previously untreated glioblastoma multiforme. Hum Gene Ther 11: 2389-2401, 2000.

7. Uckert W, Kammertöns T, Haack K, Qin Z, Gebert J, Schendel DJ and Blankenstein T: Double suicide gene (cytosine deaminase and herpes simplex virus thymidine kinase) but not single gene transfer allows reliable elimination of tumor cells in vivo. Hum Gene Ther 9: 855-865, 1998 .

8. Wolfert MA, Schacht EH, Toncheva V, Ulbrich K, Nazarova O and Seymour LW: Characterization of vectors for gene therapy formed by self-assembly of DNA with synthetic block co-polymers. Hum Gene Ther 7: 2123-2133, 1996.

9. Zhang YP, Sekirov L, Saravolac EG, Wheeler JJ, Tardi P, Clow K, Leng E, Sun R, Cullis PR and Scherrer P: Stabilized plasmid-lipid particles for regional gene therapy: formulation and transfection properties. Gene Ther 6: 1438-1447, 1999.

10. Hacein-Bey-Abina S, Von Kalle C, Schmidt M, et al: LMO2associated clonal $\mathrm{T}$ cell proliferation in two patients after gene therapy for SCID-X1. Science 302: 415-419, 2003.

11. Seggewiss R and Dunbar CE: A new direction for gene therapy: intrathymic T cell-specific lentiviral gene transfer. J Clin Invest 115: 2064-2067, 2005.

12. Puck JM and Malech HL: Gene therapy for immune disorders: good news tempered by bad news. J Allergy Clin Immunol 117: 865-869, 2006

13. Donsante A, Miller DG, Li Y, Vogler C, Brunt EM, Russell DW and Sands MS: AAV vector integration sites in mouse hepatocellular carcinoma. Science 317: 477, 2007.

14. Dave UP, Akagi K, Tripathi R, Cleveland SM, Thompson MA, Yi M, Stephens R, Downing JR, Jenkins NA and Copeland NG: Murine leukemias with retroviral insertions at $\mathrm{Lmo} 2$ are predictive of the leukemias induced in SCID-X1 patients following retroviral gene therapy. PLoS Genet 5: e1000491, 2009.

15. Yamamoto N, Hayashi Y, Kagami H, Fukui T, Fukuhara H, Tohnai I, Ueda M, Mizuno M and Yoshida J: Suicide gene therapy using adenovirus vector for human oral squamous carcinoma cell line in vitro. Nagoya J Med Sci 67: 83-91, 2005.

16. Määttä AM, Samaranayake H, Pikkarainen J, Wirth T and Ylä-Herttuala S: Adenovirus mediated herpes simplex virusthymidine kinase/ganciclovir gene therapy for resectable malignant glioma. Curr Gene Ther 9: 356-367, 2009.

17. Wang ZX, Bian HB, Yang JS, De W and Ji XH: Adenovirusmediated suicide gene therapy under the control of Cox-2 promoter for colorectal cancer. Cancer Biol Ther 8: 1480-1488, 2009.

18. Zhang Y, Yu XH, Zha X and Kong W: Adenovirus-mediated HSV-TK gene therapy using hTERT promoter in CNE cells in vitro. Chem Res Chinese U 25: 60-63, 2009.

19. Hu Z, Robbins JS, Pister A, Zafar MB, Zhang ZW, Gupta J, Lee KJ, Neuman K, Yun CO, Guise T and Seth P: A modified hTERT promoter-directed oncolytic adenovirus replication with concurrent inhibition of TGFbeta signaling for breast cancer therapy. Cancer Gene Ther 17: 235-243, 2010.

20. Song JS: Activity of the human telomerase catalytic subunit (hTERT) gene promoter could be increased by the SV40 enhancer. Biosci Biotechnol Biochem 68: 1634-1639, 2004.

21. Graham FL, Smiley J, Russell WC and Nairn R: Characteristics of a human cell line transformed by DNA from human adenovirus type 5. J Gen Virol 36: 59-74, 1977.

22. Herman JR, Adler HL, Aguilar-Cordova E, Rojas-Martinez A, Woo S, Timme TL, Wheeler TM, Thompson TC and Scardino PT: In situ gene therapy for adenocarcinoma of the prostate: A phase I clinical trial. Hum Gene Ther 10: 1239-1249, 1999.

23. Klatzmann D, Valéry CA, Bensimon G, Marro B, Boyer O, Mokhtari K, Diquet B, Salzmann JL and Philippon J: A phase I/II study of herpes simplex virus type 1 thymidine kinase 'suicide' gene therapy for recurrent glioblastoma. Study Group on Gene Therapy for Glioblastoma. Hum Gene Ther 9: 2595-2604, 1998.

24. Schellingerhout D, Rainov NG, Breakefield XO and Weissleder R: Quantitation of HSV mass distribution in a rodent brain tumor model. Gene Ther 7: 1648-1655, 2000.

25. Djeha AH, Hulme A, Dexter MT, Mountain A, Young LS Searle PF, Kerr DJ and Wrighton CJ: Expression of Escherichia coli $\mathrm{B}$ nitroreductase in established human tumor xenografts in mice results in potent antitumoral and bystander effects upon systemic administration of the prodrug CB1954. Cancer Gene Ther 7: 721-731, 2000 . 
26. Bridgewater JA, Springer CJ, Knox RJ, Minton NP, Michael NP and Collins MK: Expression of the bacterial nitroreductase enzyme in mammalian cells renders them selectively sensitive to killing by the prodrug CB1954. Eur J Cancer 31: A2362-A2370, 1995.

27. Kim NW, Piatyszek MA, Prowse KR, Harley CB, West MD, Ho PL, Coviello GM, Wright WE, Weinrich SL and Shay JW: Specific association of human telomerase activity with immortal cells and cancer. Science 266: 2011-2015, 1994.

28. Cohen SB, Graham ME, Lovrecz GO, Bache N, Robinson PJ and Reddel RR: Protein composition of catalytically active human telomerase from immortal cells. Science 315: 1850-1853, 2007.

29. Wirth T, Kuhnel F and Kubicka S: Telomerase-dependent gene therapy. Curr Mol Med 5: 243-251, 2005

30. Tang XJ, Wang YP, Zhou QH, Che GW, Chen XH and Zhu DX A study on selective killing effect of Hsv-tk/GCV driven by human telomerase catalytic subunit promoter on human lung cancer cell A549. Zhonghua Yi Xue Yi Chuan Xue Za Zhi 24: $148-152,2007$.
31. Song JS, Kim HP, Yoon WS, Lee KW, Kim MH, Kim KT, Kim HS and Kim YT: Adenovirus-mediated suicide gene therapy using the human telomerase catalytic subunit (hTERT) gene promoter induced apoptosis of ovarian cancer cell line. Biosci Biotechnol Biochem 67: 2344-2350, 2003.

32. Song J, Kim C and Ochoa ER: Sleeping Beauty-mediated suicide gene therapy of hepatocellular carcinoma. Biosci Biotechnol Biochem 73: 165-168, 2009.

33. Schepelmann S, Ogilvie LM, Hedley D, Friedlos F, Martin J, Scanlon I, Chen P, Marais R and Springer CJ: Suicide gene therapy of human colon carcinoma xenografts using an armed oncolytic adenovirus expressing carboxypeptidase G2. Cancer Res 67: 4949-4955, 2007.

34. Douin V, Bornes S, Creancier L, Rochaix P, Favre G, Prats AC and Couderc B: Use and comparison of different internal ribosomal entry sites (IRES) in tricistronic retroviral vectors. BMC Biotechnol 4: 16, 2004. 